\title{
Effet Bio-Insecticide De L'huile Essentielle Et De La Poudre De Chenopodium Ambrosioides L. Sur Les Imagos De Bruches De Haricots En Stockage
}

\author{
Mboussy Tsoungould Feldane Gladrich \\ Mpika Joseph
}

Laboratoire de biotechnologie et production végétale, Faculté des Sciences et Techniques, Université Marien Ngouabi, Brazzaville, République du Congo

\section{Mbuntcha Gueaba Hélène}

\section{Tapondjou Azefack Léon}

Unité de Recherche de Chimie Appliquée et Environnementale (URCAPE), Faculté des Sciences, Université de Dschang, Dschang, Cameroun

\section{Attibayeba}

Laboratoire de biotechnologie et production végétale, Faculté des Sciences et Techniques, Université Marien Ngouabi, Brazzaville, République du Congo

\section{Doi:10.19044/esj.2021.v17n25p267}

Submitted: 13 February 2021

Accepted: 27 May 2021

Published: 31 July 2021
Copyright 2021 Author(s)

Under Creative Commons BY-NC-ND 4.0 OPEN ACCESS

Cite As:

Mboussy Tsoungould F.G., Mpika J., Mbuntcha Gueaba H., Tapondjou Azefack L. \& Attibayeba . (2021). Effet Bio-Insecticide De L'huile Essentielle Et De La Poudre De Chenopodium Ambrosioides L. Sur Les Imagos De Bruches De Haricots En Stockage. European Scientific Journal, ESJ, 17(25), 267.

https://doi.org/10.19044/esj.2021.v17n25p267

\section{Résumé}

Le but de l'étude est d'évaluer l'effet d'insecticide et d'insectifuge de l'huile essentielle et de la poudre de Chenopodium ambrosioides L. contre Acanthoscelides obtectus Say, bruches de haricots. Après séchage de la partie terminale de $\mathrm{C}$. ambrosioides, une aliquote de la matière sèche a été utilisée pour extraire par hydro-distillation de l'huile essentielle et une autre partie broyée pour produire une poudre fine après tamisage. L'analyse de l'huile extraite a été réalisée par chromatographie en phase gazeuse à ionisation de flamme couplée à la spectrométrie de masse. La toxicité de cette huile contre les bruches a été évaluée par fumigation avec ou sans graines, répulsion, contact sur papier filtre avec graines et contact avec graines. Il a été prouvé la 
toxicité de la poudre vis à vis des bruches de haricots. Le taux de répulsion (\%) ou la mortalité des bruches a été déterminée par test. La DL50 a été calculée après que les bruches $\mathrm{A}$. obtectus ont été exposées à l'huile essentielle et à la poudre à la moitié de la durée totale de l'expérience. L'huile essentielle extraite est composée d'hydrocarbures monoterpéniques (52,14 \%) avec 37,15 $\%$ de $\alpha$-terpinène et $13,55 \%$ de $p$-cymène. Elle est riche en monoterpènes oxygénés (6,26 \%) dont l'isoascaridole représente 5,43\%. La DL50 de l'huile essentielle est de 0,0017 $\mu \mathrm{l} / \mathrm{cm} 3,0,017 \mu \mathrm{l} / \mathrm{cm} 2,0,0127 \mu \mathrm{l} / \mathrm{g}, 0,0032 \mu \mathrm{l} / \mathrm{g}$ respectivement pour le test de fumigation sans graines, de contact avec papier filtre, de fumigation avec graines et de contact avec graines. Avec la poudre, DL50 est de 19,5 g/kg. L'huile a montré un effet attractif pour le test de répulsion. Cette huile et la poudre ont influencé le cycle de développement des bruches en réduisant leur nombre des imagos émergées en F1. Cette huile a été plus efficace que la poudre. Le test in planta confirmera l'efficacité de l'huile essentielle extraite de C. ambrosioides contre les bruches A. obtectus.

Mots-clés : Insectifuge, Insecticide, Chromatographie En Phase Gazeuse, Spectrométrie De Masse, Tests De Toxicité, Acanthoscelides Obtectus

\section{Bio-Insecticide Effect Of Essential Oil And Powder Of Chenopodium Ambrosioides L. On Imagos Of Beans In Stocking}

\section{Mboussy Tsoungould Feldane Gladrich Mpika Joseph}

Laboratoire de biotechnologie et production végétale, Faculté des Sciences et Techniques, Université Marien Ngouabi, Brazzaville, République du Congo

\section{Mbuntcha Gueaba Hélène}

\section{Tapondjou Azefack Léon}

Unité de Recherche de Chimie Appliquée et Environnementale (URCAPE),

Faculté des Sciences, Université de Dschang, Dschang, Cameroun

\section{Attibayeba}

Laboratoire de biotechnologie et production végétale, Faculté des Sciences et Techniques, Université Marien Ngouabi, Brazzaville, République du Congo

\section{Abstract}

The aim of this study is to evaluate the insecticide and insectifuge effect of Chenopodium ambrosioides L. essential oil and powder against Acanthoscelides obtectus Say which are beans' weevil. After drying the terminal part of C. ambrosioides, a certain quantity of dried latter was used for extraction by hydro-distillation of essential oil and the other part was grounded 
to produce a thin powder sifting. Analysis of the extracted oil was done by chromatography in gaseous phase at flame ionization coupled with mass spectrometry. Toxicity of the oil on weevil was evaluated by fumigation with or without seeds, repulsion, contact on filter paper with seeds and contact with seeds. It was proven the toxicity of the powder face to beans' weevil. Repulsion rate (\%) or mortality of the weevils was determined by test. DL50 was calculated after weevil A. obtectus have been exposed to essential oil and powder at half time of the total duration of the experimentation. The extracted essential oil is composed of monoterpenic hydrocarbons (52.14\%) with 37.15 $\%$ of $\alpha$-terpinen and $13.55 \%$ of p-cymene. The oil is rich in oxygenated monoterpenes (6.26 \%) of which the isoascaridol represents $5.43 \%$. The DL50 of the essential oil equals $0.0017 \mu \mathrm{l} / \mathrm{cm} 3,0.017 \mu \mathrm{l} / \mathrm{cm} 2,0.0127 \mu \mathrm{l} / \mathrm{g}, 0.0032$ $\mu \mathrm{l} / \mathrm{g}$ for the fumigation without seeds test, the contact with filter paper, fumigation with seeds and contact with seeds respectively. With the powder, the DL50 equals $19.5 \mathrm{~g} / \mathrm{kg}$. The oil showed an attractive effect for the test of repulsion. That oil and the powder influenced the development cycle of the weevils by reducing the number of images emerged in F1. That oil was more efficient than the powder. The test in planta will confirm the efficiency of the extracted essential oil from $\mathrm{C}$. ambrosioides against the weevils $A$. obtectus.

Keywords: Insectifuge, Insecticide, Chromatography In Gazeous Phase, Mass Spectrometry, Toxicity Tests, Acanthoscelides Obtectus

\section{Introduction}

La conservation de denrées vivrières est confrontée à diverses problèmes phytosanitaires liés aux contraintes biotiques telles que les insectes, les champignons et les rongeurs et abiotiques telles que la température, humidité... (Divy et al., 2015). Les agressions des insectes ravageurs sur la culture de Phaseolus vulgaris L. (haricot commun) provoquent des pertes de production et des dégâts considérables dans les biotopes tropicaux humides (Buycks, 1962 ; Azouma, 1990). Ainsi, les pertes provoquées par ces insectes sont très importantes, en raison des facteurs climatiques très favorables à leur croissance et le type d'entreposage peu propice à la lutte contre les bruches en stock (Caswell, 1968). A la République du Congo, l'espèce Acanthoscelides obtectus (Say) (Coleoptera; Bruchidae) est le principal insecte nuisible aux stocks de la culture de haricot commun (Delobel et Epouna-Mouinga, 1984).

Parailleurs, cette bruche est aussi présente dans d'autres pays africains comme l'Algérie, le Cameroun, la République Centrafricaine (RCA), la République Démocratique du Congo (RDC)... (Tapondjou et al., 2002 ; Kaloma et al., 2008 ; Ndomo et al., 2009 ; Goucem, 2017). En effet, elle cause des dégâts non négligeables en plantation et en stockage (Singh et al, 1990 ; Goucem, 2014). En Algérie, les pertes annuelles du haricot stocké dû à 
l'infestation par Acanthoscelides obtectus (Say) ont été estimées entre 7 et 73\% (Bouchikhi et al., 2010). L'utilisation d'insecticides ou fumigènes de synthèse est l'une des méthodes de lutte efficace contre ces ravageurs (Haubruge et al., 1988). Le produit utilisé entraîne souvent un effet résiduel néfaste sur la santé humaine et la biocénose. Il perturbe des niches écologiques et pollue l'environnement (Sun et Davidson, 1998 ; Chemat et al., 2012). En conséquence, les insecticides de bonne qualité sont rares et chers en milieu rural.

Actuellement, diverses études se focalisent sur les plantes présentant des propriétés insecticides, insectifuges ou anti-appétantes vis à vis des insectes ravageurs des produits entreposés (Tapondjou et al., 2002 ; Kaloma et al., 2008 ; Ndomo et al., 2009 ; Goucem, 2017 ; Messaoudene et Mouhou, 2017). Ces propriétés ont conduit à la découverte des métabolites secondaires actifs chez certaines espèces végétales (Ocimum basilicum, Cymbopogon citratus, Nicotina tabacum, etc. ). Cependant, les études menées sur la protection de denrées stockées ont montré une efficacité différentielle spécifique des huiles essentielles extraites ou des poudres de certaines plantes contre des insectes (Tapondjou et al., 2002 ; Kaloma et al., 2008; Messaoudene et Mouhou, 2017). L'usage des plantes dans la protection de denrées stockées contre les ravageurs est une pratique ancienne très répandue en Afrique et en Asie (Delobel et Malonga, 1987 ; Seck et al., 1996 ; Kaloma et al., 2008). Afin de protéger les denrées stockées en milieu rural, les paysans congolais de Brazzaville mélangent les semences avec la cendre de bois ou avec des organes séchés des plantes odorantes tels que des feuilles Chenopodium ambrosioides L., Lippia multiflora L., Nicotiana tabacum L. et les rhizomes de Zingiber officinale Rosc. (Delobel et Malonga, 1987; Mboussy, 2019). Mais, l'effet insecticide et insectifuge de l'huile essentielle et de la poudre de la partie terminale de Chenopodium ambrosioides L. sur les imagos de bruches de haricot en stockage in vitro est mal connu. En effet, il n’a été réalisée aucune étude au Congo-Brazzaville abordant spécifiquement cette problématique. De ce qui précède, nous postulons l'hypothèse que l'huile essentielle et la poudre de la partie terminale de Chenopodium ambrosioides L. sur les imagos de bruches de haricots en stockage in vitro ont un effet insecticide et /ou insectifuge. L'objectif de cette étude est d'évaluer les effets insecticides et insectifuges d'huile essentielle et de la poudre de la partie terminale de Chenopodium ambrosioides sur les imagos de Acanthoscelides obtectus en stockage in vitro. 


\section{Materiel Et Methodes \\ Matériel}

Matériel animal, élevage en masse et bio tests et matériel végétal

Pour le matériel animal, les imagos A. obtectus (Say) ont été collectées auprès des vendeurs de Haricot commun sur le marché $« \boldsymbol{B} »$ de la ville de Dschang à l'Ouest de Cameroun. Ces imagos ont été entretenues pour faire un élevage de masse dans l'enceinte de l'Unité de Recherche de Chimie Appliquée et Environnementale (URCAPE) de l'Université de Dschang. Concernant l'élevage en masse et bio tests, la variété de haricot petit grain rouge (Vigna umbellata (Thrub.) Ohwi et Ohshi) a été choisie pour l'élevage en masse et pour les tests de toxicité.

Pour le matériel végétal, les organes utilisés, étaient les parties terminales de Chenopodium ambrosioides pour l'extraction d'huile essentielle et la poudre. Ces parties terminales étaient composées des feuilles de rameaux florifères, des boutons floraux, des fleurs épanouies et des graines de $C$. ambrosioides. Sur la plante de C. ambrosioides, les parties terminales ont été récoltées en décembre 2019 et en janvier de 2020 au sein du Campus de l’Université de Dschang et à ses alentours à l'Ouest du Cameroun.

\section{Méthodes}

Elevage de masse, préparation de la poudre, extraction d'huile essentielle, analyse et identification des composés de l'huile essentielle

Pour un élevage de masse, les insectes adultes tous sexes confondus de Acanthoscelides obtectus obtenus chez des commerçants du marché « B » de la ville de Dschang. Ces insectes ont été utilisés pour infester les grains de haricot contenus dans les bocaux en verre de 3 litres. Dans un bocal, il a été introduit en moyenne 1000 insectes pour $1200 \mathrm{~g}$ de haricot. Ces bocaux ont été conservés à une température moyenne de $24,98 \pm 1,2^{\circ} \mathrm{C}$ et une humidité relative de $67,15 \pm 1,15 \%$.

Concernant la préparation de la plante pour l'extraction d'huile essentielle et l'obtention de la poudre, après la récolte, les parties terminales de Chenopodium ambrosioides ont été transportées au Laboratoire de Chimie Appliquée et Environnementale pour séchage. Avant le séchage, ces parties terminales ont été triées pour retirer les débris inertes (poussières...) et vivants (Nématodes, Gastropodes, Insectes, Fourmis). Elles ont été d'abord défeuillées puis découpées en petits morceaux. Le séchage s'est effectué pendant 5 jours à une température moyenne de $25,04 \pm 1,19{ }^{\circ} \mathrm{C}$ et une humidité relative moyenne de 57,96 $\pm 0,27 \%$. Le brassage a été effectué trois fois par jour afin d'assurer une meilleure aération et homogénéité du séchage afin d'éviter l'apparition des moisissures et des pourritures.

Après le séchage de la partie terminale de $C$. ambrosioides, un premier aliquote a servi à l'extraction de l'huile essentielle. Cette huile a été extraite 
par hydrodistillation à l'aide d'un appareil de type Clevenger. L'huile essentielle obtenue a été déshydratée avec du sulfate de sodium anhydre puis conservée à $4^{\circ} \mathrm{C}$ au réfrigérateur jusqu'à son utilisation. L'analyse de l'huile essentielle obtenue a été effectuée par une chromatographie de type Agilent 6890 couplée à un spectromètre de masse 5973N et un détenteur à l'ionisation à flamme et équipé d'une colonne HP INNOWax avec deux injecteurs de 30 $\mathrm{m}$ de longueur, $250 \mu \mathrm{m}$ de diamètre et $0,25 \mu \mathrm{m}$ d'épaisseur de film de phase stationnaire de type polyéthylène glycol. Ce chromatogramme est couplée à un spectromètre de masse HP 5972 dans les conditions d'analyse suivantes :

- $\quad$ la température du four a été maintenue à $250{ }^{\circ} \mathrm{C}$, à une vitesse de 5 ${ }^{\circ} \mathrm{C} / \mathrm{min}$;

- $\quad$ gaz vecteur : hélium $(1,2 \mathrm{ml} / \mathrm{min})$;

- $\quad$ la température du détecteur a été maintenue à $250^{\circ} \mathrm{C}$, le flux d' $\mathrm{H}_{2}$ à 40 $\mathrm{ml} / \mathrm{min}$ et flux d'air à $450{ }^{\circ} \mathrm{C}$. La détection des masses a été réalisée en mode balayage entre 32 et 450 Daltons.

Deux signaux sont enregistrés correspondant à la spectrométrie de masse et au détecteur à ionisation à flamme permettant respectivement d'identifier et quantifier les molécules. L'identification des différents composés a été faite à partir de leurs spectres de masse et de leurs indices de rétention polaire dans la phase stationnaire en comparaison avec ceux contenu dans la bibliothèque interne du Laboratoire Central d'Analyse de l'Entreprise Marne en France, commerciale ou répertoriés dans la lecture. La quantification de chaque composé a été effectuée par intégration de son pic sur le spectre du chromatographe en phase gazeuse.

Un second aliquote des parties terminales de $C$. ambrosioides séchées a été broyé à l'aide d'un broyeur électrique (Jankle and Kundel KG, Typ A10, N5614, Allemagne) pour obtenir la poudre. Le broyat a été tamisé à l'aide d'un tamis de maille de $0,5 \mathrm{~mm}$ de diamètre pour donner une poudre fine à particules de granulométrie homogène.

\section{Evaluation de l'effet insectifuge ou répulsif de l'huile essentielle}

Ce test a permis de déterminer le pourcentage de répulsion d'huile par la méthode de la zone préférentielle sur papier filtre Wathman (HPL.3.1) décrite par Mc Donald et al. (1970). Des disques de papier filtre de $9 \mathrm{~cm}$ de diamètre ont été coupés en deux parties égales (31,80 $\mathrm{cm}^{2}$ chacune). Quatre solutions ont été préparées avec des volumes respectifs de 0,$5 ; 1 ; 2$ et $4 \mu \mathrm{l}$ d'huile essentielle diluées respectivement dans l'acétone $0,5 \mathrm{ml}$. Chacune des solutions obtenues a été répartie uniformément sur l'un de demi-disque du papier filtre. L'autre moitié du papier a reçu uniquement $0,5 \mathrm{ml}$ de l'acétone servant de témoin. Après 10 minutes d'évaporation complète de l'acétone, les concentrations respectives de 0,$015 ; 0,031 ; 0,062$ et $0,125 \mu \mathrm{l} / \mathrm{cm}^{2}$ de surface ont été obtenues par les demi-disques traités par la solution (huile essentielle 
+ acétone). Les deux demi-disques ont été rassemblés par une bande adhésive transparente et placés dans les boîtes de pétri. Ensuite, 20 imagos Acanthoscelides obtectus, de sexe confondus, âgés de deux jours au plus ont été disposées au centre de chaque boîte de pétri. Quatre répétitions ont été réalisées pour chaque volume de chacune de ces huiles. Les boîtes à pétri ont été placées sur la palliasse à une température moyenne de $25,75 \pm 0,43^{\circ} \mathrm{C}$ avec une humidité relative moyenne de 40,25 $\pm 0,82 \%$. Au bout deux heures, il a été réalisé le dénombrement des insectes présents sur chaque demi-disque dans les boites. Le pourcentage de répulsion a été calculé.

\section{Toxicité d'huile essentielle par fumigation ou inhalation avec ou absence des graines}

Toxicité par fumigation ou inhalation en absence des graines

Dans des bocaux en verre de $270 \mathrm{~cm}^{3}$ de volume à couvercle étanches, 20 imagos de Acanthoscelides obtectus de sexes confondus et âgées de trois jours au plus ont été introduites dans chacun des bocaux. Des carrés de papier filtre (Whatman HPL3.1) de $4 \mathrm{~cm}^{2}$ de surface ont été fixés chacun à l'aide d'un fil noir au centre de la face interne des couvercles afin qu'ils prennent la mi-hauteur de ces bocaux. Des volumes des huiles essentielles $(0,5 ; 1 ; 2$ et 4 $\mu \mathrm{l})$ dilués à $0,1 \mathrm{ml}$ d'acétone ont été répandues sur ces carrés de papier filtre. Après 20 minutes, temps nécessaire pour l'évaporation complète d'acétone, les concentrations respectives de 0,$002 ; 0,004 ; 0,007$ et $0,015 \mu \mathrm{l} / \mathrm{cm}^{3}$ d'huile essentielle ont été obtenues au sein de ces bocaux. Parallèlement, le témoin ayant reçu uniquement $0,1 \mathrm{ml}$ d'acétone. Chaque traitement a été répété quatre fois. L'ensemble des tests a été réalisé à une température moyenne de 26,5 \pm $1,66{ }^{\circ} \mathrm{C}$ et l'humidité relative $60 \pm 6,04 \%$ pendant 24 heures. Le dénombrement des insectes morts a été effectué après 6,12 et 24 heure dans chaque bocal. Il a été déterminée le pourcentage mortalité par concentration de l'huile essentielle testée.

Toxicité par fumigation ou inhalation en présence des graines Dans chacun des bocaux en verre de $270 \mathrm{~cm}^{3}$ de volume à couvercle étanches, il a été introduit $50 \mathrm{~g}$ de grains de haricot rouge et 20 imagos de sexes confondus, âgées de trois jours. Des carrés de papier Whatman HPL3.1. de $4 \mathrm{~cm}^{2}$ de surface ont été fixés chacun à l'aide d'un fil noir au centre de la face interne des couvercles afin qu'ils prennent la mi-hauteur de ces bocaux. Les préparations des solutions (huile essentielle + acétone) ont été faites avec différents volumes des huiles essentielles $(0,5 ; 1 ; 2$ et $4 \mu \mathrm{l})$ diluée $0,1 \mathrm{ml}$ d'acétone. Ainsi, $10 \mu \mathrm{l}$ de chaque solution d'huile ont été répandues sur des carrés de papier correspondant ainsi aux concentrations respectives de 0,002 ; 0,$004 ; 0,007$ et $0,015 \mu \mathrm{l} / \mathrm{cm}^{3}$ d'huile essentielle dans les bocaux après évaporation complète d'acétone. Les carrés de papier filtre dans les bocaux témoins ayant reçu uniquement l’acétone, quatre répétitions ont été réalisées 
pour chaque traitement. Ce test a été réalisé à une température moyenne de $24,66 \pm 1,54{ }^{\circ} \mathrm{C}$ et l'humidité relative $49 \pm 3,60 \%$ pendant 24 heures. Le dénombrement des insectes morts a été effectué après 6,12 et 24 heure dans chaque bocal. Le pourcentage mortalité a été calculé en fonction de la durée d'exposition et de la concentration par concentration de l'huile essentielle.

\section{Toxicité d'huile essentielle par contact sur papier filtre ou graines de haricots \\ Toxicité d'huile essentielle par contact sur papier filtre}

La toxicité par contact sur papier filtre des huiles essentielles a été évaluée en utilisant les concentrations respectives de $0 ; 0,007 ; 0,015$; 0,031 et $0,062 \mu \mathrm{l} / \mathrm{cm}^{2}$ par surface de papier filtre. Ces différentes concentrations ont été obtenues en diluant les volumes respectifs d'huile essentielle de $0 ; 0,5 ; 1 ; 2$ et $4 \mu \mathrm{l}$ dans $1 \mathrm{ml}$ d'acétone qui sont répandues pour chacune sur des disques de papier filtre Whatman (HPL3.1) de $9 \mathrm{~cm}$ de diamètre (soit $63,6 \mathrm{~cm}^{2}$ de surface). Après 20 minutes, temps d'évaporation complète d'acétone, insectes adultes âgés de deux jours au plus ont été introduits dans des boites de pétri de $9 \mathrm{~cm}$ de diamètre. Quatre répétitions ont été effectuées pour toutes les concentrations. Ces tests ont été réalisés à une température moyenne de $25 \pm 2,45{ }^{\circ} \mathrm{C}$ et l'humidité relative 42,25 $\pm 1,92 \%$ pendant 48 heures. Les mortalités des bruches ont été dénombrées toutes les 12 heures pendant les deux jours d'exposition. Le pourcentage mortalité a été déterminé selon la concentration par concentration de l'huile essentielle testée.

\section{Toxicité par contact d'huile essentielle sur graines}

Dans les boîtes en verre de $270 \mathrm{~cm}^{3}$ de volume, il a été pesé $50 \mathrm{~g}$ de graines de haricot. Ces graines ont été traitées par quatre doses d'huile essentielle préparées comme précédemment en dissolvant les volumes de 0,5 ; 1 ; 2 et $4 \mu \mathrm{l}$ de d'huile essentielle dans $1 \mathrm{ml}$ d'acétone, correspondant aux doses respectives de 0,$01 ; 0,02 ; 0,04$ et $0,08 \mu \mathrm{l} / \mathrm{g}$ par graines après 5 min d'agitation pour homogénéisation et évaporation complète d'acétone. Il a été déposé 20 imagos âgées de deux jours au plus dans chaque bocal en verre et recouvert d'un tissu poreux. Le lot témoin a été traité uniquement à l'acétone. Quatre répétitions ont été effectuées pour toutes les doses. L'ensemble des bocaux en verre a été placé dans une enceinte à une température moyenne de 26,25 $\pm 1,5$ ${ }^{\circ} \mathrm{C}$ et l'humidité relative $53 \pm 1,41 \%$. Les imagos mortes ont été dénombrées toutes les 12 heures au bout de 2 jours. Après le dénombrement, le pourcentage mortalité a été estimée par concentration de l’huile essentielle testée. 


\section{Toxicité par contact de la poudre de Chenopodium ambrosioides sur graines de haricots}

Il a été introduit $50 \mathrm{~g}$ de graines de haricot dans les boîtes en verre de $270 \mathrm{~cm}^{3}$ de volume. Ces graines ont été traitées par quatre doses de poudre des masses de 0,$025 ; 0,05 ; 0,1$ et $0,2 \mathrm{~g}$ de la poudre de Chenopodium ambrosioides, correspondant aux doses respectives de 0,$05 ; 0,1 ; 0,2$ et $0,4 \% / \mathrm{g}$ par graines après 5 min d'agitation pour homogénéisation. Dans chaque bocal en verre, il a été déposé 20 imagos âgées de deux jours au plus ensuite recouvert d'un tissu poreux. Le lot témoin a été traité uniquement à l'acétone. Quatre répétitions ont été effectuées pour toutes les doses. L'ensemble des bocaux en verre a été placé dans une enceinte à une température moyenne de $25,75 \pm 0,5^{\circ} \mathrm{C}$ et l'humidité relative $68,5 \pm 2,5 \%$. Les imagos mortes ont été dénombrées toutes les 24 heures pendant 4 jours et le pourcentage mortalité déterminé selon la dose de la poudre.

\section{Impact de l'huile essentielle ou poudre de Chenopodium ambrosioides sur l'émergence de la première génération de Acanthoscelides obtectus (Say)}

L'impact de l'huile ou la poudre de Chenopodium ambrosioides sur l'émergence de la première génération de Acanthoscelides obtectus a été évalué dans les bocaux en verre. Il a consisté à compter le nombre d'imagos émergées sur les graines de Vigna umbellata traitées par différentes doses d'huile essentielle ou poudre. Pour cela, à l'issu des tests de toxicité par contact sur graines des différents traitements, les bocaux ont été maintenu $\mathrm{s}$ dans les mêmes conditions pendant 15 jours. Au bout de ce laps temps, toutes imagos mortes ou vivantes ont été débarrassées des bocaux en verre par tamisage. A la suite de cette opération, les bocaux ont été conservés dans les mêmes conditions expérimentales jusqu'à émergence de la première génération. Le dénombrement d'imagos émergées a été effectué tous les jours jusqu'à la fin d'émergence de la F1. Il a été calculé le pourcentage de réduction.

\section{Appréciation de la mortalité des insectes}

Un insecte a été considéré comme mort lorsqu'un insecte a été trouvé en décubitus dorsal ou ventral, le corps raide. Il ne présentait aucune réaction surtout au niveau des pattes et des antennes après stimulation au moyen d'une pince à bruches.

\section{Variables mesurées et calculées}

Trois variables ont été mesurées à l'intervalle régulier après l'incubation des boites ou des bocaux : insectes présents sur chaque demidisque dans une boite de Pétri, insectes morts dénombrés pour la toxicité de l'huile essentielle et poudre, et les imagos émergées de Acanthoscelides 
obtectus sur les graines après leur contact avec l'huile essentielle ou la poudre de Chenopodium ambrosioides.

Pour l'effet insectifuge ou répulsif de l'huile essentielle, le dénombrement des insectes présents sur chaque demi-disque dans les boites a été réalisé et le pourcentage de répulsion (PR) a été calculé en utilisant la formule de Mc Donald et al. (1970):

$$
\operatorname{PR}(\%)=\stackrel{\mathrm{Nc}-\mathrm{Nt}}{\mathrm{Nc}+\mathrm{Nt}} \times 100
$$

Nc : nombre d'insectes présents sur la partie du disque traité uniquement à l'acétone ; Nt : nombre d'insectes présents sur la partie du disque traité avec la solution (acétone + huile essentielle)

Le pourcentage moyen de répulsion pour chaque dose d'huile essentielle vis-à-vis de Acanthoscelides obtectus a été calculé et attribué à l'une des différentes classes répulsives variant de 0 à 5 comme indiqué dans le tableau 1 (MC Donald et al., 1970).

Tableau 1. Classement de la répulsivité des huiles essentielles selon Mc Donald et al. (1970)

\begin{tabular}{|c|c|}
\hline Classe & Intervalle de répulsion \\
\hline 0 & $\mathrm{PR} \leq 0,1 \%$ \\
\hline I & $0,1<\mathrm{PR} \leq 20 \%$ \\
\hline II & $20,1<\mathrm{PR} \leq 40 \%$ \\
\hline III & $40,1<\mathrm{PR} \leq 60 \%$ \\
\hline IV & $60,1<\mathrm{PR} \leq 80 \%$ \\
\hline V & $80,1<\mathrm{PR} \leq 100 \%$ \\
\hline
\end{tabular}

Concernant la toxicité de l'huile essentielle et de la poudre de Chenopodium ambrosioides, les insectes morts dénombrés dans un bocal ont permis de déterminer le pourcentage de mortalité selon la concentration et la durée d'exposition. Les pourcentages dans les bocaux traités ont été exprimés par la formule d'Abbott (1925) en pourcentage de mortalité corrigée (MC), en tenant compte du pourcentage de mortalité naturelle enregistré dans les bocaux témoins (Mt) :

$$
\mathbf{M C}=\frac{\begin{array}{l}
\text { Mo }-\mathrm{Mt} \\
100-\mathrm{Mt}
\end{array}}{10100}
$$

et la méthode de Bliss (1938) citée par Ndomo (2006) et Woguem (2017) a été utilisée pour déterminer la CL50 (équation 1) tel que décrite cidessous : 
Soit $\mathbf{y}=\mathbf{a x}+\mathbf{b}$ (1) l'équation de la droite de régression définie par l'équation:

$$
\begin{aligned}
& \mathrm{x}=\frac{\mathrm{y}-\mathrm{b}}{\mathrm{a}} \quad \begin{array}{l}
\mathrm{x}=\log \text { (concentration) } ; \\
\mathrm{y}=\text { probit de mortalité } ;
\end{array} \\
& \text { où } \quad \mathrm{a}=\text { pente de la droite ; } \\
& \mathrm{b}=\text { intersection de la droite de régression } \\
& \text { avec l'axe des ordonnées }
\end{aligned}
$$

Pour concentration $=\mathrm{CL}_{50}$, on $\mathrm{a}:$

$$
\log C_{50}=\frac{y-b}{a} \longrightarrow C_{50}=10^{\frac{y-b}{a}}
$$

Après contact des graines avec l'huile essentielle ou la poudre de Chenopodium ambrosioides, les imagos de Acanthoscelides obtectus émergées ont permis de calculer le pourcentage de réduction de l'émergence. Le pourcentage de réduction (PR) ou d'inhibition (PI) a été calculé par la formule décrite par Tapondjou et al. (2002) :

$$
\text { PR ou PI }=\frac{\mathrm{CN}-\mathrm{TN}}{\mathrm{CN}} \times 100
$$

D’où CN : nombre d'imagos émergées dans les bocaux de contrôles ; TN : nombre d'imagos émergées dans les bocaux traités

\section{Analyses statistiques}

Le logiciel XLSTAT version 7.5.3 a été utilisé. Après vérification de la normalité de distribution des résidus et celle de l'homogénéité des variances des sous populations analysées, la comparaison de moyennes des variables mesurées a été réalisée par le test-Student Newman et Keuls au seuil de risque de $5 \%$. Le modèle linéaire s'écrit $\mathrm{Y}=\mathrm{Y}=\mu+\mathrm{T}+\varepsilon$. Où, $\mathrm{Y}$ est la variable réponse; $\mu$ la moyenne générale; $\mathrm{T}$, représente le traitement; $\varepsilon$, l'erreur ; $\varepsilon i j$ est supposé être normalement distribué avec une moyenne nulle et une variance $\sigma 2$, c'est à dire, $\varepsilon i j \sim N(0, \sigma 2)$.

\section{Resultats}

\section{Rendement d'extraction d'huile essentielle extraite de la partie terminale de chenopodium ambrosioides}

L'huile essentielle collectée était de couleur jaune pâle avec une odeur forte persistante. Elle a été obtenue avec un rendement de 1, $06 \%$ par rapport à la matière sèche utilisée. Pour la composition chimique, cette huile essentielle était constituée de 13 composés représentant 58,2 \% des composés identifiés regroupés en hydrocarbures monoterpèniques $(52,14 \%)$ et 
monoterpènes oxygénés $(6,26 \%)$. Les composés majoritaires sont $\alpha$-terpinène (37,15\%), P-cymène (13,55\%) et Isoascaridole (5,43 \%) $(\mathrm{T}$ ableau 2). En effet, la classe d'hydrocarbures monoterpèniques est la plus représentée avec $52,14 \%$ en comparaison de celle des monoterpènes oxygénés (6,26 \%).

Tableau 2. Principaux composés d'huile essentielle de la partie terminale de Chenopodium ambrosioides L

\begin{tabular}{|c|c|c|}
\hline Composés identifiés & $\mathbf{0 *}^{*}$ & IR $^{*}$ \\
\hline Hydrocarbures monoterpènique & $\mathbf{5 2 , 0 0 2}$ & \\
\hline Myrcène & 0,058 & 1166 \\
\hline --terpinène & $\mathbf{3 7 , 1 5 7}$ & 1192 \\
\hline Limonène & 0,5 & 1206 \\
\hline Beta phellandrène & 0,108 & 1217 \\
\hline Gamma terpinène1 & 0,567 & 1253 \\
\hline P-cymène & $\mathbf{1 3 , 5 5}$ & 1280 \\
\hline Terpinolène & 0,062 & 1290 \\
\hline Monoterpènes oxygénés & $\mathbf{6 , 1 9 1}$ \\
\hline Cis p-menthadien-2,8-ol-1 & 0,1 & 1624 \\
\hline P-cymen-8-ol & 0,1 & 1847 \\
\hline Ascaridole & 0,255 & 1865 \\
\hline Isoascaridole & $\mathbf{5 , 4 3 3}$ & 1872 \\
\hline Thymol & 0,1 & 2176 \\
\hline Carvacrol & 0,203 & 2204 \\
\hline
\end{tabular}

\section{Légende}

\%* : Pourcentage. IR* : Indice de rétention

\section{Effet insectifuge d'huile essentielle de Chenopodium ambrosioides}

Le tableau 3 présente l'effet répulsif de l'huile essentielle issue de la partie terminale de Chenopodium ambrosioides vis-à-vis des imagos de Acanthoscelides obtectus après deux heures d'exposition. Les résultats ont révélé une propriété attractive de l'huile essentielle testée vis-à-vis des imagos de A. obtectus. Cet effet attractif augmente avec la diminution de la concentration en huile.

Tableau 3. Répulsion (\%) sur papier filtre d'huile essentielle de la partie terminale de

Chenopodium ambrosioides L. vis-à-vis des Imagos de Acanthoscelides obtectus (Say)

\begin{tabular}{|c|c|}
\hline Concentration $\left(\boldsymbol{\mu l} / \mathbf{c m}^{2}\right)$ & Taux de répulsion (\%) \\
\hline 0,015 & $-22,50 \pm 09,57$ \\
\hline 0,031 & $-20,00 \pm 37,41$ \\
\hline 0,062 & $-50,00 \pm 25,81$ \\
\hline 0,125 & $-37,50 \pm 27,53$ \\
\hline
\end{tabular}

Effets fumigants de l'huile essentielle de Chenopodium ambrosioides à l'égard des imagos de Acanthoscelides obtectus.

Cette activité a été évaluée avec ou sans graines. La figure 1 illustre les pourcentages de mortalités cumulées et corrigées (\%) des imagos de 
Acanthoscelides obtectus en fonction de la durée d'exposition et des concentrations d'huile essentielle. Ces taux croissent avec l'augmentation des concentrations de l'huile essentielle. La concentration la plus forte $(0,015$ $\mu \mathrm{l} / \mathrm{cm}^{3}$ ) a provoqué $100 \%$ de mortalité au bout de 6 heures d'exposition. A l'opposé, chez les témoins, une mortalité naturelle de 12,5 \% a été enregistrée au bout de 12 heures d'exposition. Ainsi, la valeur $\mathrm{CL}_{50}\left(0,0017 \mu \mathrm{l} / \mathrm{cm}^{3}\right)$ calculée après 12 heures d'exposition atteste l'efficacité de toxicité de cette huile essentielle par fumigation sans graines vis-à-vis de ces imagos.

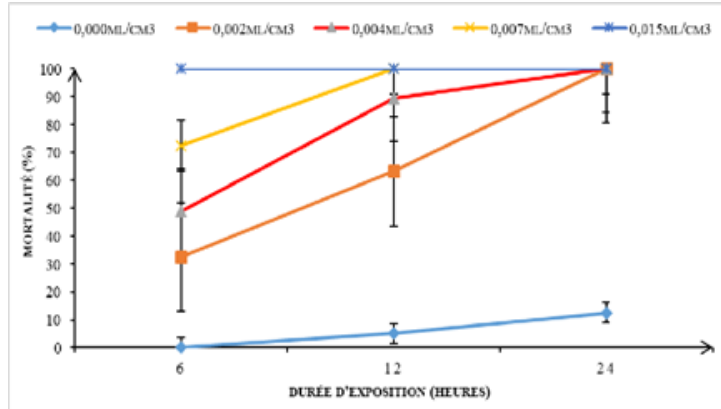

Figure 1. Evolution du pourcentage de mortalités cumulées des imagos de Acanthoscelides obtectus (Say) par fumigation sur papier filtre soumis aux différentes concentrations d'huile essentielle de Chenopodium ambrosioides L. pendant 24 heures

La figure 2 illustre les mortalités cumulées (\%) des imagos de Acanthoscelides obtectus dans des graines de haricot traitées à différentes doses en fonction de la durée d'exposition. Il en ressort que le pourcentage de mortalité des imagos de A. obtectus croît en fonction de la dose d'huile dans les graines pendant la durée d'exposition. La plus forte dose $(0,08 \mu \mathrm{l} / \mathrm{g})$ a provoqué $100 \%$ de mortalité des imagos en 24 heures d'exposition. La faible valeur $\operatorname{DL}_{50}(0,0127 \mu \mathrm{l} / \mathrm{g})$ calculée après 12 heures d'exposition atteste l'efficacité de toxicité de cette huile essentielle par fumigation sur graines visà-vis de ces imagos. Aucune mortalité n’a été notée dans les bocaux témoins.

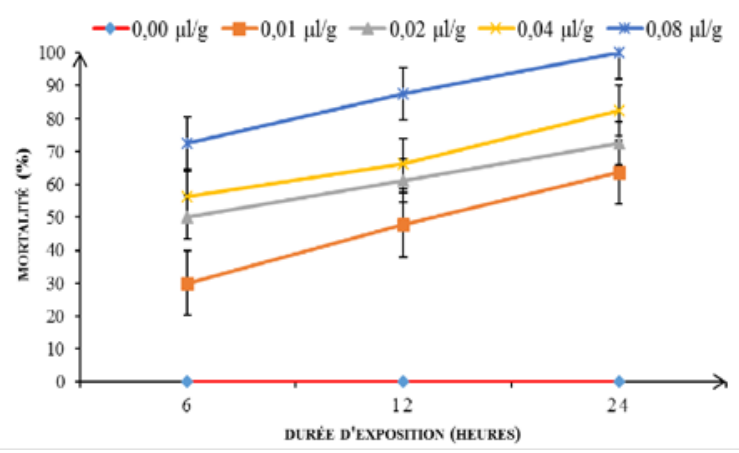

Figure 2. Evolution du pourcentage de mortalités cumulées des imagos de Acanthoscelides obtectus (Say) par fumigation avec graines soumises aux différentes doses de l’huile essentielle de Chenopodium ambrosioides L. pendant 24 heures 


\section{Toxicité par contact de l'huile essentielle de Chenopodium ambrosioides L. sur des imagos de Acanthoscelides obtectus}

Cette toxicité a été évaluée par contact sur papier filtre et sur les graines de haricot traitées à différentes doses d'huile essentielle. La figure 3 montre que les mortalités cumulées et corrigées (\%) des imagos de A. obtectus varient selon les concentrations d'huile essentielle utilisées pour traiter les papiers filtre. La plus forte dose $\left(0,062 \mu \mathrm{l} / \mathrm{cm}^{2}\right)$ a provoqué $100 \%$ de mortalité des imagos au bout 12 heures d'exposition. La faible valeur DL50 $\left(0,017 \mu \mathrm{l} / \mathrm{cm}^{2}\right)$ calculée en 24 heures d'exposition atteste l'efficacité de toxicité de cette huile essentielle vis-à-vis de ces imagos.

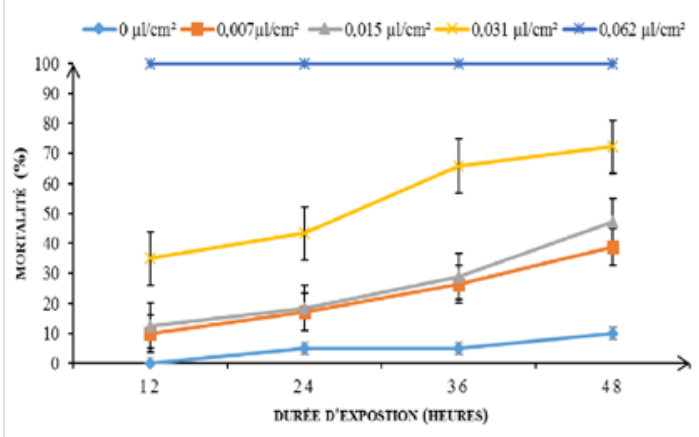

Figure 3. Evolution des pourcentages de mortalité des imagos de Acanthoscelides obtectus (Say) par contact sur papier filtre soumis à différentes concentrations d'huile essentielle de Chenopdium ambrosioides L. en fonction du temps

La figure 4 illustre les pourcentages de mortalités cumulées (\%) des imagos de Acanthoscelides obtectus en fonction de la durée d'exposition. Ils ont varié selon les doses d'huile essentielle utilisées pour traiter les graines. La dose de $0,08 \mu \mathrm{l} / \mathrm{g}$ a provoqué $90 \%$ de mortalité des imagos au bout 12 heures d'exposition et $100 \%$ de mortalité des imagos au bout 48 heures. Aucune mortalité n'a été notée dans les bocaux témoins. La faible valeur DL50 $(0,0032 \mu \mathrm{l} / \mathrm{g})$ calculée en 24 heures d'exposition atteste l'efficacité de toxicité de cette huile essentielle vis-à-vis de ces imagos.

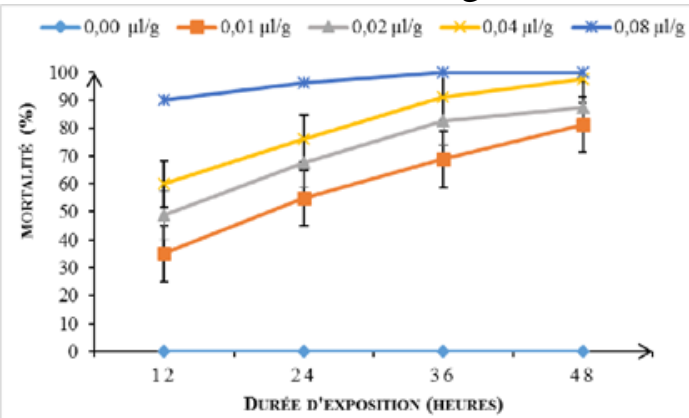

Figure 4. Evolution des pourcentages de mortalité des imagos de Acanthoscelides obtectus (Say) par contact sur les graines traitées avec différentes doses d’huile essentielle de Chenopodium ambrosioides L. en fonction du temps. 


\section{Toxicité par contact sur graines traitées avec la poudre de Chenopodium ambrosioides vis-à-vis des imagos de Acanthoscelides obtectus.}

La figure 5 illustre les mortalités cumulées (\%) des imagos de Acanthoscelides obtectus en fonction de la durée d'exposition. Ces mortalités ont varié selon les doses de la poudre utilisées pour traiter les graines. Il était noté une mortalité de 47,5\% sur les imagos en contact avec la plus forte dose $(0,4 \%)$ au bout de 96 heures d'exposition. La faible valeur DL50 (1, 95 \% soit 19,5g/Kg de graines) calculée en 48 heures d'exposition, atteste l'efficacité de toxicité de cette huile poudre vis-à-vis de ces imagos.

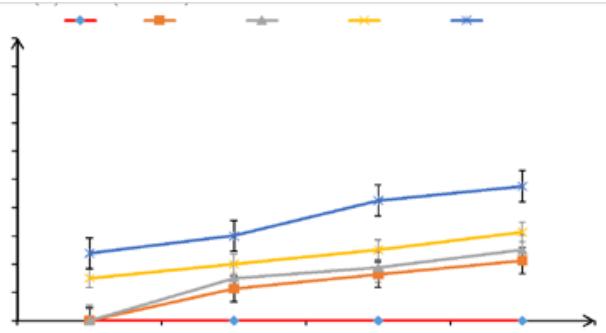

Figure 5. Evolution des pourcentages de mortalité des imagos de Acanthoscelides obtectus (Say) par contact avec les graines traitées différentes doses de la poudre de Chenopodium ambrosioides en fonction du temps

\section{Impact de l'huile essentielle et de la poudre sur l'émergence de la première génération $\left(F_{1}\right)$}

Les tableaux 4a et $4 \mathrm{~b}$ présentent les effets de l'huile essentielle et la poudre de Chenopodium ambrosioides sur l'inhibition de l'émergence de la $\mathrm{F}_{1}$ des imagos de Acanthoscelides obtectus. Il en ressort que le nombre d'imagos émergées est fonction de la dose d'huile essentielle et de la poudre de $C$. ambrosioides utilisées. Il a été enregistré 42 et 77 des imagos émergées respectivement dans les bocaux témoins de l'huile essentielle et de la poudre avec $0 \%$ de réduction. Par contre, une inhibition de la nouvelle génération a été observée dans les bocaux traités avec l'huile essentielle et la poudre. A la dose $0,08 \mu \mathrm{l} / \mathrm{g}$ de graines inhibe la nouvelle génération et la dose $0,4 \% / \mathrm{g}$ de graines a enregistrée une réduction de $74,51 \%$.

Tableau 4a. Impact des doses d'huile essentielle sur l'émergence de la première génération

$\left(\mathrm{F}_{1}\right)$ et de réduction de l'émergence des imagos de Acanthoscelides obtectus (Say)

\begin{tabular}{|c|c|c|}
\hline Dose $(\boldsymbol{\mu l} / \mathbf{g})$ & $\begin{array}{c}\text { nombre des imagos } \\
\text { émergées à la } \mathbf{F}_{\mathbf{1}}\end{array}$ & $\begin{array}{c}\text { \% de réduction de l'émergence } \\
\text { des imagos à la } \mathbf{F}_{\mathbf{1}}\end{array}$ \\
\hline 0 & $42,00 \mathrm{a} \pm 7,61$ & 0 \\
\hline 0,01 & $25,25 \mathrm{~b} \pm 1,70$ & 39,88 \\
\hline 0,02 & $14,50 \mathrm{c} \pm 1,29$ & 65,47 \\
\hline 0,04 & $11,50 \mathrm{c} \pm 1,91$ & 72,61 \\
\hline 0,08 & $0,00 \mathrm{~d} \pm 0,00$ & 100 \\
\hline
\end{tabular}

$\%$ : Pourcentage 
Tableau 4b. Impact des doses de poudre essentielle sur l'émergence de la première génération $\left(\mathrm{F}_{1}\right)$ et de réduction de l'émergence des imagos de Acanthoscelides obtectus (Say)

\begin{tabular}{|c|c|c|}
\hline Dose (\%/g) & $\begin{array}{c}\text { nombre des imagos } \\
\text { émergées à la } \mathbf{F}_{\mathbf{1}}\end{array}$ & $\begin{array}{c}\text { \% de réduction de l'émergence } \\
\text { des imagos à la } \mathbf{F}_{\mathbf{1}}\end{array}$ \\
\hline 0 & $77,50 \mathrm{a} \pm 52,10$ & 0 \\
\hline 0,05 & $75,25 \mathrm{a} \pm 34,32$ & 2,90 \\
\hline 0,1 & $46,50 \mathrm{a} \pm 35,70$ & 40 \\
\hline 0,2 & $22,25 \mathrm{a} \pm 9,94$ & 71,29 \\
\hline 0,4 & $19,75 \mathrm{a} \pm 14,86$ & 74,51 \\
\hline
\end{tabular}

$\%$ : Pourcentage

\section{Discussion}

\section{Test in vitro pour la vérification de l'efficacité de la conservation des graines.}

L'huile essentielle et la poudre de Chenopodium ambrosioides ont été testées in vitro pour leur efficacité dans la conservation des graines de haricot vis-à-vis des imagos de Acanthoscelides obtectus. L'extraction de l'huile essentielle a rapporté un rendement de $1,06 \%$ sur la partie terminale de $C$. ambrosioides composée des feuilles et des extrémités fleuries. Ces résultats sont analogues à ceux obtenues par Tapondjou et al. (2003) et El et al. (2016). Ces auteurs ont noté un rendement d'extraction des huiles essentielles de 0,8\% sur les feuilles séchées $C$. ambrosioides respectivement au Cameroun et au Maroc. Ainsi, la couleur jaune et l'odeur forte de cette l'huile seraient dues à la présence du Myrcène. L'analyse chimique de l'huile essentielle extraite a montré qu'elle est composée majoritairement de $\alpha$-terpinène $(37,15 \%)$, Pcymène (13,55\%) et Isoascaridole (5,43\%). Elhourri et al. (2016) ont été observée une différence de teneur sur ces deux composés principaux : $\alpha$ terpinène $(38,9 \%)$ et $\mathrm{P}$-cymène (26,4\%). Tapondjou et al (2003) ont noté des teneurs de $50 \%$ de $\mathrm{P}$-cymène et 37,6\% $\alpha$-terpinèn. Par contre, Owolabi et al. (2009) ont obtenu $63,1 \%$ de $\alpha$-terpinène et 3,6\% Isoascaridole. De telles variations quantitatives et qualitatives du rendement et de la composition chimique sont dues aux facteurs extrinsèques et intrinsèques (Nguimale, 2016). A cela s'ajoute les conditions de réalisation de l'extraction et la méthode d'analyse de la composition chimique. L'ascariole est le principal composé de l'huile essentielle de $C$. ambrosioides sa teneur varie entre 40 à 75\% (Malloy,1923; Smile, 1924 ; Dembistk et al. 2008). Or, $\alpha$-terpinène est le précurseur d'ascaridole par biotransformation enzymatique au sein de cette plante (Johnson, 1984). L'analyse chimique d'huile extraite a montré une forte teneur en $\alpha$-terpinène $(37,15 \%)$ et une faible teneur en ascaridole $(0,25 \%)$. Ceci s'expliquerait par le fait que lors de la récolte de cette espèce végétale, elle n'avait pas encore achevé sa biosynthèse. Sur les plants de $C$. ambrosioides, la stimulation de la synthèse de $\alpha$-terpinène renforcera son effet bioinsecticide pour une lutte contre les bruches de haricots en stockage. 


\section{Les Tests de toxicité}

Les tests de toxicité avec l'huile essentielle de C. ambrosioides révèlent une forte mortalité des bruches de haricots. Cette mortalité varie de manière significative en fonction de la dose appliquée et la durée d'exposition. Pour l'huile essentielle de C. ambrosioides, il a été enregistré $100 \%$ de mortalité avec la concentration de $0,015 \mu \mathrm{l} / \mathrm{cm}^{3}$ en 24 heures d'exposition au test par fumigation avec graines. La dose $0,08 \mu \mathrm{l} / \mathrm{g}$ de cette huile a occasionné 100\% de mortalité en 48 heures d'exposition au test par contact avec graines. La toxicité de cette huile essentielle vis-à-vis des imagos de A. obtectus peut être expliquée aux composés majoritaires $\alpha$-terpinène $(37,15 \%)$, P-cymène $(13,55 \%)$ et Isoascaridole $(5,43 \%)$. Ces résultats sont similaires à ceux de Tapondjou et al. (2002 ; 2003). Ces derniers ont montré l'efficacité de l’huile essentielle des feuilles de $C$. ambrosioides L. vis-à-vis des imagos de Callosobruchus maculatus, Acanthoscelides obtectus, Callosobruchus chinensis, Stitophilus granariuns, Stitophilus zea maïs et Prostephanus truncatus. Mais, les résultats observés sont contraires aux études antérieures réalisées par Malloy (1923) ; Smile et Pessoa (1924) et Dembistk et al. (2008). Pour ces auteurs, la toxicité de l'huile essentielle de $C$. ambrosioides serait due à l'ascaridole, représentant 40 à 75\% à lui seul. L'ascaridole possède un principe actif, qui est très toxique pour les animaux à sang froid, paralyse et tue les vers parasitaires (Dembistk et al. 2008). L'analyse chimique d'huile essentielle extraite a montré une faible teneur en ascaridole (0,25\%). Ceci s'expliquerait que lors de la récolte de cette espèce végétale, elle n’avait pas encore achevé sa biosynthèse. La forte toxicité de l'huile essentielle n'est pas uniquement due l'ascaridole mais aussi aux composés majoritaires $\alpha$ terpinène, p-cymène, Isoascaridole. Cette action peut aussi découler des effets synergides des composés minoritaires tels que limonène qui est avéré toxique vis-à-vis de diverse bruches de denrées stockées (Regnault-Roger et Hamraoui, 1995; Prates et al. 1998 ; Fogang Dongmo, 2013). Les résultats sont similaires à ceux de Tapondjou et al. (2003), qui mentionnent cette faible teneur d'ascaridole dans l'huile essentielle de feuilles de C. ambrosioides L. La toxicité de l'huile essentielle de C. ambrosioides vis-à-vis des imagos de A. obtectus constituerait un volet de lutte intégrée contre les bruches de haricots.

\section{Effet insectifuge d'huile essentielle}

Pour l'effet insectifuge d'huile essentielle de C. ambrosioides, il a été noté le taux de répulsion négatif. Cette huile essentielle extraite a présenté un effet attractif vis-à-vis des imagos de $A$. obtectus. Ces résultats sont contraires à ceux de Tapondjou et al. (2003). Pour ces auteurs, l'huile essentielle issue des feuilles de $C$. ambrosioides L. a montré un effet répulsif vis-à-vis des imagos de C. maculatus. Ceci s'expliquerait par l'absence ou l'inhibition des 
composés répulsifs par l'existence d'autres composés dans cette huile essentielle extraite ou par la présence des composés attractifs tels que le myrcène ou encore par la sensibilité de la bruche (Casida, 1990 ; Cardona et Karel, 1990). Woguem (2012) a mentionné qu'une huile essentielle pourrait posséder à la fois un effet répulsif ou attractif sur les insectes ravageurs. Comme l'huile essentielle issue des fruits de Xylopia parviflora (A. Rich.) Benth. a montré un effet attractif et répulsif respectivement vis-à-vis des imagos de $A$. obtectus et $C$. maculatus.

\section{Efficacité de la poudre}

La poudre de C. ambrosioides s'est révélée efficace contre les imagos d'A. obtectus. La dose $0,4 \%$ de poudre par gramme de graines a provoqué 47,5\% de mortalité au bout de 96 heures d'exposition par le test de contact sur graines. L'efficacité de la poudre est analogue à celle obtenue par Tapondjou et al. (2003). Pour ces auteurs, la poudre de feuilles de C. ambrosioides L. a provoqué une mortalité de 52\% vis-à-vis des imagos de Callosobruchus maculatus au bout de 96 heures d'exposition. Cette différence pourrait s'expliquer par le fait de la sensibilité de la bruche et l'organe végétal testé. Au Congo-Brazzaville, Delobel et Malonga (1987) ont montré que la poudre de $C$. ambrosioides possède des effets insecticides considérables contre Caryedon serratus de Arachis hypogaea. D’autres études menées par Delobel et Epouna-Mouinga (1984) ont approuvé que l'association des feuilles de $C$. ambrosioides et des graines de Phaseolus vulgaris en stock en milieu paysan protégeait ces plantes contre les agressions des A. obtectus. L'étude réalisée par Kaloma et al. (2008) a montré que les poudres de Eucalyptus citriodora, de Cuperssus lucitanica et de Tagetas minitiflora en RDC ont été efficaces pour assurer la conservation des graines de Zea mays et de Phaseolus vulgaris.

\section{Effet bio-insecticide de l'huile essentielle et de la poudre}

Les résultats obtenus ont montré une réduction ou une inhibition du nombre des imagos émergées de $A$. obtectus à la première descendance après le traitement en huile essentielle et en poudre de $C$. ambrosioides. Une inhibition complète a été observée sur les graines traitées avec la dose de 0,8 $\mu \mathrm{l} / \mathrm{g}$ de l'huile essentielle, cela indiquerait que les imagos ayant subi ce traitement avaient eu une courte période de survie, qui ne leur a pas permis de réaliser l'oviposition d'où l'absence de la première génération. Ensuite, pour les graines traitées avec les doses faibles, les imagos ont survécu pendant une durée importante. Ce qui apermis de réaliser une oviposition faible, réduisant la descendance de la première génération par rapport aux témoins. La dose de $0,4 \% / g$ de graines a provoqué $74,51 \%$ de réduction de l'émergence de la nouvelle descendance. L'huile essentielle et la poudre de C. ambrosioides L. pourraient avoir un impact sur l'oviposition et la survie des œufs de $A$. 
obtectus, elles ont réduit ou inhibé l'émergence de la première génération. Ces résultats sont similaires à ceux de Tapondjou et al. (2002) et de nombreux autres auteurs Ndomo (2006) ; Woguem (2017) ; Pegalepo et al. (2019). Au Cameroun, les études ont montré que l'huile essentielle et la poudre de $C$. ambrosioides ont une activité inhibitrice sur les imagos de $A$. obtectus et $C$. maculatus (Tapondjou et al., 2002). Cela semblerait dû à la présence de terpènes qui ont des effets inhibiteurs de la reproduction des insectes. Pollack et al. (1993) ; Kiuschi et al. (2002) et Monzote et al. (2006) ont en effet montré que l'ascaridole possède un potentiel d'inhibition in vivo du développement de Plasmodium falciparum, Trypanosoma cruzi, Luschmania amazonensis. Daoudi et al. (2017) ont montré que le C. ambrosioides possède une activité fongique contre Aspergillus brasiliensis, Alternaria sp. et Fusarium oxysporum.

\section{Conclusion}

La présente étude a révélé l'efficacité de l'huile essentielle et la poudre de la partie terminale de chenopodium ambrosioides L. dans la protection du haricot en stock à l'égard des bruches. En effet, l'huile essentielle et la poudre de $C$. ambrosioides se sont montrées toxiques et réduisent d'émergence des imagos en $\mathrm{F}_{1}$ en fonction des doses utilisées in vitro. Elle a aussi mis en évidence une propriété attractive ces imagos. Cependant, cette huile est plus active par rapport à sa poudre. Ce travail vient de confirmer les connaissances endogènes de la population congolaise sur la phytoprotection de cette denrée en stock. Cette essence et sa poudre peuvent être utilisées comme substances premières actives dans la formulation des phytoinsecitides. Les activités sont poursuivies afin de trouver une formulation spécifique nécessaire aux essais au champ.

\section{Remerciements}

Cette étude a été financée par l'Agence Universitaire de la Francophonie (AUF) et l'Organisation des Nations Unies pour l'Alimentation et l'Agriculture (FAO). Nous exprimons la profonde gratitude au Professeur Léon AZEFACK TAPONDJOU et son équipe pour avoir accueilli dans leur Unité de Recherche de Chimie Appliquée et Environnementale (URCAPE).

\section{References:}

1. Abbott W.S., 1925. A method of computing the effectiveness of an insecticide. Journal of Economic Entomology. 18 : 265-267.

2. Alzouma I., 1990. Les problèmes de la post-récolte en Afrique sahélienne. In: Fouabi K. and Philogene J. (eds.). Actes du Séminaire International de la post-récolte en Afrique. Abidjan, Côte d'Ivoire. 29 jan. - 02 fev. 22-27. 
3. Bliss C., 1939. The determination of the dosage-mortality curve from small numbers. Quarterly Journal of pharmacology.11: 192-216.

4. Bouchikhi T. Z., Bendahou M., Khelil M. A., 2010. Lutte contre les bruches Acanthoscelides obtectus et la mite Tineoba bisselliella par les huiles essentielles extraites de deux plantes aromatiques d'Algérie. Lebanese, Science journal. $11: 1$.

5. Buycks E. J. L., 1962. Précis des maladies et insectes nuisibles rencontrés sur les plantes cultivées au Congo, au Rwanda et au Burundi. Bruxelles, p. 601.

6. Cardona C. \& Karel A.L., 1990. Key insects and other invertebrate pests of beans. In: Singh S.R. (ed.). Insect Pests of Tropical Food Legumes. IITA Ibadan, Nigeria. John Wiley and Sons Ltd. pp. 157191.

7. Casida J.H., 1990. Pesticide mode of action, evidence for implications of a finite number of biochemical targets. In: Casida J.E. (ed.). Pesticides and alternatives. Innovative chemical and Biological Approaches to Pest Control. Amsterdam: Elsevier, pp: 11-22.

8. Caswell G. H., 1968. The storage of cowpea in Northerm States of Nigeria. Proceedings of the Agriculture Society of Nigeria. (5): 4-5.

9. Chemat F., Vian A.M., Cravotto G., 2012. Green extraction of natural products: Concept and principles. Int. J. Mol. Sci., 13 : 8615-8627.

10. Daoudi A., Bammou M., Haloui Z., Ibijbijen J., Nassiri L., 2017. Activité antifongique des extraits aqueux de Cadendula officinalis L., Urginea maritima (L.) Baker et Chenopodium ambrosioides L. European Scientific Journal. 13 (24), 483-497.

11. Delobel A., Epouna-Mouinga, 1984. La bruche Acanthoscelides obtectus et la commercialisation du haricot sur le Plateau Koukouya (District de Lekana). Republique du Congo, 17 p.

12. Delobel A., Malonga P., 1987. Insecticide properties of six plant materials against Caryedon serratus (OL) (Coleoptera; Bruchdae). Journal Stored Products Research, 23: 173-176.

13. Dembitsk V., Shkrob I., Hanus L.O., 2008. AScaridole and related peroxidesfrom the Genus Chenopodium. Biomed Pap Med Fac Uni Palacky Olomouc Czech Repub. 152 (2): 209-215.

14. Diby Y. K. S., Tahiri Y. A., Akpesse A. A. M., Tra Bi C. S., 2015. Graine : haricot. In: Agriculture en Afrique Tropical, Bruxelles, DGCI : 337-355.

15. El I. M., Elhourri M., Amechrouq A., Lemrhari A., Belmalha S., Echchgadda G., 2016. Caractérisation chimique de l'huile essentielle de Chenopodium ambrosioides (L.) (Chenopodiaceae) de quatre régions du Maroc. J. Master. Environ. Sci. 7(11): 4087-4095. 
16. Fogang Dongmo H. P., 2013. Utilisation des huiles de quatre plantes aromatiques camerounaises dans la préservation de la santé humaine en exploitant leurs propriétés insecticides, antiradicalaires, cytoyoxiques et antibactériennes. Mémoire de Doctorat, Faculté des Sciences, Université de Dschang, Cameroun, 166p.

17. Goucem Khelfane K. (2014). Etude de l'activité insecticide des huiles essentielles et des poudres de quelques plantes à l'égard de la bruche du haricot, Acanthoscelides obtectus Say (Coléoptère ; chrysomelidae, Bruchudae) et comportement de ravageurs vis-à-vis des composés volatils de différentes variétés de la plante hote (Phaseolus vulgaris L.) Thèse doctorat. Université Mouloud MAMMERI de Tizi-Ouzou, $144 p$.

18. Haubruge E., Shiffers B., Gabriel E. \& Verstraeten C., 1988. Etude de la relation dose-efficacité de six insecticides à l'égard de Sitophilus granarius L., S. oryzae L., S. zeamais Mots. (Coleoptera, Curculionidae). Mededelingen Faculteit Landbouwwetenschappen Rijksuniversiteit Gent. 53/2b, 719-726.

19. Johnson M. A., 1984. Biosynthesis of ascaridole. Arch Biochem Biophys. 235: 254-6.

20. Kaloma A., Kitambala K., Ndjango N.L., Sinzahera U., Paluku T., 2008. Effet des poudres d'Eucalyptus citriodora, de Cupressus lucitanica et de Tagetas minitiflora dans la conservation du maïs (Zea mays) et du haricot (Phaseolus vulgaris) dans les conditions de Rethy (République Démocratique du Congo). Tropicultura, 1: 24-27.

21. Kiuchi F., Itano Y., Uchiyama N., Honda G., Tsubouchi A., NakajimaShimada J., Aoki T., 2002. Monterpene hydroperoxides with trypanocidal from Chenopodium ambrosioides. Journal Natural Product. 65: 509-512.

22. Malloy D. M., 1923. Pharmacology and therapeutics of oil of Chenopodium and investigation on the anthelmintic value of its components. Journal Pharmacology, 24 : 391-400.

23. Mboussy Tsoungould F. G., 2019. Evaluation des rendements des huiles essentielles des plantes a effet insecticide ou insectifuge sur les ravageurs de haricots et pois au Congo. Mémoire de Master, Faculté des Sciences et Techniques, Université Marien Ngouabi, Brazzaville, République du Congo, 36p.

24. McDonald L.L., Guy R.H., Speirs R.D., 1970. Preliminary evaluation of new candidate materials as toxicants, repellents and attractants against stored product insects. Marketing Res. Rep. $\mathrm{n}^{\circ} 882$. Washington: Agriculture Research Service, US Departement of Agriculture, $183 \mathrm{p}$. 
25. Messaoundene H., Mouhou N., 2007. Etude de la toxicité des huiles essentielles contre les ravageurs des denrées stockées. Etude de la toxicité des huiles essentielles contre les ravageurs des denrées stockées. Mémoire de Doctorat, Faculté des Sciences de la Nature et la Vie, Université Abderrahmane MIR-Bejaia, Algérie, 35p.

26. Monzote L., Montalvo A.M., Almanonni S., Scull R., Miranda M. Abreu J., 2006. Activity of the essential oil from Chenopodium ambrosioides grown in Cuba against Leischmania amazonensis. Chemotherapy. 52 : 130-136.

27. Ndomo A. F., 2006. Evaluation de l'effet insecticide de formulation poudreuse de Callistemon viminalis (Wild.) J. D. Hookex. Benth. et Callistesmon viminalis W. à l'égard des adultes de Acanthoscelides obtectus (Say) et Callosobruchus maculatus (Fab.) Mémoire de Master, Faculté des Sciences, Université de Dschang, Cameroun, 74p.

28. Ndomo A. F., Tapondjou A.L., Tendonkeng F., Tchouanguep F.M., 2009. Evaluation des propriétés insecticides des feuilles de Callistemon viminalis (Myrtaceae) contre les adultes d'Acanthoscelides obtectus (Say) (Coleoptera ; Bruchidae). Tropicultura. 27 (3): 137-143.

29. Nguimale K. B. T., 2016. Evaluation de l'activité insecticide de l'huile essentielle de Citronelle (Variété géante Lemon Grass) contre les larves de Desmestes sp. Mémoire de Master, Faculté des Sciences et Techniques, Université Marien Ngouabi, Brazzaville, Congo, 36p.

30. Owolabi S. M., Ladjide L., Oladimeji O. M., Setzer N. W., Palazzo C. M., Olowu A. R., Ogundajo A., 2007. Volatile Contituents and Antibacterial Screening of the Essentiel Oil of Chenopodium ambrosioides L. growing in Nigeria. Natural Product Communications. 4(7): 989-992.

31. Pegalepo F., Dossa Sedami S. G., Bokonon-Ganta A. H., Dossou J., 2019. Effet de la poudre de Piper guineense Schumach \& Thonn., 1827. (Piperaceae) sur les paramètres semenciers et la mortalité de Sitophilus oryzea L., 1953 (Coleoptera : Curculionidea) dans le stock des semences de riz. European Scientific Journal. 15 (15), 190-205.

32. Pollack Y., Segal R., Golenser J, 1993. The effect of ascaridole on the in vitro developpement of Plasmodium falciparum. Parasitol Research.76: 570-572.

33. Prates H.T., Santos J.P., Waquil J.M., Fabris J.D., Oliveira A.B. \& Foster J.E., 1998. Insecticidal activiy of monoterpenes against Rhyzoperta dominica (F.) and Tribolium castaneum (Herbst.). Journal of Stored Products Research.34 (4): 243-249. 
34. Regnault-Roger C. \& Hamraoui A., 1995. Efficiency of plants from South of France used as traditional protectants of Phaseolus vulgaris L. Journal of Stored Products Research.29 (3): 259-264.

35. Seck D., Lognay G., Haubruge E., Marlier M., Gaspar C., 1996. Alternative protection of cospeas seeds against Callosobruchus maculatus (F.) (Coleoptera: Bruchidae) using hermetic storage alone or in combination with Boscia senegalensis (Pers.), Lam. Ex Poir. Journal Stored Products Research.32 (1): 39-44.

36. Singh S.R., Jakai L.E.N., Dos Santos J.H.R. \& Adalla C.B., 1990, Insect pests of cowpea. In: Singh S.R., Van Emden H.F. \& Taylor J.A., (eds.) Pest of grain legumes: ecology and control, London/New-York, Academic press, pp. 219-229

37. Smilie W. G., Pessoa S. B., 1924. A study of the anthelmintic properties of the constituents of the oil of Chenopodium. Journal Pharmacology. 24: 359-70.

38. Sun W. Q., Davidson P., 1998. Protein inactivation in amorphous sucrose and trehalose matrices: effects of phase separation and crystallization. Biochimica et Biophysica Acta. 1425 : 235-244.

39. Tapondjou L. A., Adler C., Bouda H., Fontem D. A. 2003. Bioefficacité des poudres et des huiles essentielles des feuilles de Chenopodium ambrosioides et Eucalyptus saligna à l'égard de bruche du niébé, Callosobruchus maculatus Fab. (Coleoptera, Bruchidae). Cahiers Agricultures. 12 (6) : 401-407.

40. Tapondjou L. A., Adler C., Bouda H., Fontem, D. A., 2002. Efficacy of powder and essential oil from Chenopodium ambrosioides leaves as post-harvest grain protectants against six-stored product beetles. Journal of Stored Products Research. (38) : 395-4.

41. Woguem V., 2012. Caractérisation chimique et evaluation des propriétés insecticides des huiles des fruits de Piper capense L. (Piperaceae) et Xylopia parviflora (A. Rich.) Benth (Anonnaceae) à l'égard des adultes de Acanthoscelides obtectus (Say). et Callosobruchus maculatus (Fab.) (Bruchidea). Thèse de Master, Université de Dschang, Cameroun, Dschang, Faculté des Sciences, Biochimie, $70 \mathrm{p}$.

42. Woguem V., 2017. Etude des activités insecticides, antifongicides, antioxydantes, antiproliférative et caractérisation chimique des huiles essentielles de cinq plantes aromatiques du Cameroun. Thèse de doctorat, Université de Dschang, Cameroun, Dschang, Faculté des Sciences, Biochimie, $140 \mathrm{p}$. 